\title{
Microdureza de resina composta: efeito de aparelhos e tempos de polimerização em diferentes profundidades
}

\section{Microhardness of composite resins: effect of photocuring units and polymerization periods in different depths}

\author{
Luciana Aily SANTOS* \\ Míriam Lacalle TURBINO** \\ Michel Nicolau YOUSSEF*** \\ Edmir MATSON****
}

\begin{abstract}
SANTOS, L. A.; TURBINO, M. L; YOUSSEF, M. N.; MATSON, E. Microdureza de resina composta: efeito de aparelhos e tempos de polimerização em diferentes profundidades. Pesq Odont Bras, v. 14, n. 1, p. 65-70, jan./mar. 2000.

As propriedades das resinas compostas têm sido estudadas com freqüência, bem como os fatores que podem influenciar seu grau de polimerização. Diante da evolução desses materiais e da necessidade de buscarmos melhora do seu comportamento na cavidade bucal, objetivamos, por meio deste estudo avaliar a eficácia de dois aparelhos fotopolimerizadores do tipo pistola (de alta intensidade de luz), comparando com a de um aparelho a cabo (de baixa intensidade de luz), com tempos de exposição de 20 e de 40 segundos e em profundidades de 1 a 4 milímetros. Os testes avaliaram o grau de polimerização da resina por meio de testes de microdureza Knoop. Os resultados mostraram haver diferença estatisticamente significante entre os tempos, sendo que com 40 segundos a dureza foi maior que com 20 segundos para as 4 diferentes profundidades. Para o fator aparelhos, os dois aparelhos tipo pistola se comportaram superiores ao do tipo cabo Fibralux (Dabi Atlante), e entre eles, o XL 1500 (3M) promoveu dureza maior que o Optilight II (Gnatus) no tempo de polimerização de 40 segundos. As profundidades de 1, 2, 3 e 4 milimetros mostraram estatisticamente diferença entre si tendo sido encontrada maior dureza para as menores profundidades $(\mathrm{p}<0,05)$.
\end{abstract}

UNITERMOS: Resinas compostas; Materiais dentários; Dureza.

\section{INTRODUÇÃO}

Estando a Odontologia distante de um material ideal para substituir a perda parcial ou total do órgão dental, busca-se eliminar propriedades negativas e aumentar as positivas dos materiais existentes.

Por isso, as propriedades das resinas compostas têm sido reavaliadas com freqüência, bem como os fatores que nelas podem influenciar.

Sabe-se, também, que o grau de polimerização pode alterar essa dureza e depende de vários fatores como: tempo de fabricação ou tempo de prateleira do produto, tipo de aparelho fotopolimerizador $^{16,19}$, cor da resina, tempo de polimerização ${ }^{5,15}$, tempo pós-polimerização ${ }^{4}$, intensidade da luz e profundidade em resina composta ${ }^{9,10,12,17,18}$.

A profundidade de polimerização pode também ser afetada pela distância entre a ponta do fotopolimerizador e a restauração, ocorrendo a diminui- ção da intensidade de luz conforme a espessura da resina composta ${ }^{2,14}$.

Um adequado comprimento de onda deve ser alcançado em toda área da restauração, para o máximo de polimerização e sucesso clínico a longo prazo $^{17}$.

Quanto à intensidade de luz, os primeiros aparelhos fotopolimerizadores produziam um baixo fluxo luminoso (menos de $200 \mathrm{~mW} / \mathrm{cm}^{2}$ ), pois perdiam luz ao longo do trajeto, tinham constantes fraturas das microfibras, deterioravam o espelho dicróico, fatores esses que interferiam negativamente. Por isso, esses aparelhos foram substituídos pelos de pistola ou revólver, onde a lâmpada é posicionada próxima à ponta de saída da luz.

BARGHI et al. ${ }^{3}$ (1994) concluíram que $30 \%$ de um total de 205 unidades fotopolimerizadoras usadas em consultório particular forneciam menos que $200 \mathrm{~mW} / \mathrm{cm}^{2}$, um valor considerado como inadequado para uma perfeita polimerização.

\footnotetext{
* Pós-graduanda, nível Mestrado; ** Professora Doutora; *** Professor Associado; **** Professor Titular - Departamento de Dentística da Faculdade de Odontologia da Universidade de São Paulo.
} 
SANTOS, L. A.; TURBINO, M. L; YOUSSEF, M. N.; MATSON, E. Microdureza de resina composta: efeito de aparelhos e tempos de polimerização em diferentes profundidades. Pesq Odont Bras, v. 14, n. 1, p. 65-70, jan./mar. 2000.

Com base nesses achados, o objetivo desta pesquisa foi comparar o grau de dureza Knoop da resina composta através de corpos-de-prova, nas profundidades de 1, 2, 3 e $4 \mathrm{~mm}$, em tempos de 20 e $40 \mathrm{~s}$, em 3 aparelhos fotopolimerizadores, 2 do tipo pistola e 1 a cabo.

\section{MATERIAL E MÉTODO}

Foram utilizados três aparelhos fotopolimerizadores no qual dois deles, o Optilight II (Gnatus) e o XL 1500 (3M) eram tipo revólver e o aparelho Fibralux (Dabi Atlante) era a cabo; todos operando em 110 volts. Testamos dois tempos de polimerização (20 e 40 s) da resina composta $Z 100$ (3M), na cor A2; quatro profundidades $(1,2,3$ e $4 \mathrm{~mm}$ ) e três repetições com três medidas para cada região.

Para os testes, utilizamos uma matriz de poliacetato composta de 2 partes: uma totalmente plana e outra contendo uma cavidade retangular de $6 \mathrm{~mm}$ de profundidade, $4 \mathrm{~mm}$ de largura e $3 \mathrm{~mm}$ de altura, já descrita por TURBINO et al. ${ }^{19}$ (1992).

A cavidade da hemimatriz foi preenchida com a resina e coberta com uma lamínula de vidro para promoção de maior lisura superficial e ausência de bolhas. A seguir, era sobreposta a outra metade da matriz, ficando apenas uma pequena extremidade em contato com o meio externo, na qual fazia-se a incidência de luz do fotopolimerizador por tempos de 20 e de $40 \mathrm{~s}$.

Terminada a polimerização, a parte superior da matriz e a lamínula de vidro eram removidas. A superficie da resina a ser analisada era aquela que havia estado em contato com a lamínula.

Com bisturi e régua, milimetrou-se essa super- fície, marcando espaços de $1 \mathrm{~mm}$ nos 4 primeiros milimetros de profundidade.

A resina composta, logo após sua polimerização e ainda posicionada na hemimatriz, era levada ao microdurômetro HVM2000 (Shimadzu) com o auxílio de uma morsa universal ajustável (Shimadzu) para prender o corpo-de-prova e posicionar a superficie a ser examinada paralela ao plano horizontal da base do aparelho. Para os testes foi utilizado o penetrador Knoop com 50 g de carga, pelo tempo de $45 \mathrm{~s}$. Em cada milimetro, eram realizados 3 ensaios de dureza nos $2 \mathrm{~mm}$ centrais de cada profundidade.

Todos os valores obtidos foram submetidos à analise estatística.

\section{RESULTADOS}

Os resultados obtidos consistiram de 216 valores de dureza Knoop. Para análise estatística foram realizadas as médias das três medidas, resultando num total de 72 valores (Tabela 1). Esses dados foram avaliados com auxílio do software GMC 7.0 desenvolvido pelo Prof. Dr. Geraldo Maia Campos da Fac. Odont. de Ribeirão Preto - USP.

Os testes preliminares mostraram que os dados amostrais tiveram uma distribuição normal e homogênea, o que possibilitou a utilização da Análise de Variância (Tabela 2). Os resultados dessa análise mostraram haver diferença estatisticamente significante para os 3 fatores avaliados.

Para o fator tempo $(F=6,78)$, constatou-se que com $40 \mathrm{~s}(\mathrm{~m}=48,30)$ a microdureza foi maior que com $20 \mathrm{~s}(\mathrm{~m}=41,72)$.

Para o fator aparelho $(F=144,0)$, o aparelho a

TABELA 1 - Valores de Microdureza - dados originais.

\begin{tabular}{l|c|c|c|c|c|c|c|c}
\hline \hline \multirow{4}{*}{} & \multicolumn{2}{|c|}{$1 \mathrm{~mm}$} & \multicolumn{2}{c|}{$2 \mathrm{~mm}$} & \multicolumn{2}{c}{$3 \mathrm{~mm}$} & \multicolumn{2}{c}{$4 \mathrm{~mm}$} \\
\cline { 2 - 9 } & $20 \mathrm{~s}$ & $40 \mathrm{~s}$ & $20 \mathrm{~s}$ & $40 \mathrm{~s}$ & $20 \mathrm{~s}$ & $40 \mathrm{~s}$ & $20 \mathrm{~s}$ & $40 \mathrm{~s}$ \\
\hline \multirow{4}{*}{ Optilight II } & 58,40 & 63,13 & 57 & 52,20 & 43,70 & 50,66 & 30,83 & 45,33 \\
\cline { 2 - 9 } & 65,43 & 64,43 & 61,43 & 60,20 & 55,43 & 54,93 & 44,70 & 42 \\
\cline { 2 - 9 } & 73,30 & 56,10 & 65,96 & 49,56 & 57,66 & 44,06 & 42,03 & 38,56 \\
\hline \multirow{4}{*}{ XL 1500 } & 61,66 & 79,47 & 62 & 75,66 & 55,16 & 66,33 & 40,20 & 57,50 \\
\cline { 2 - 9 } & 73,30 & 72,70 & 69,20 & 69,23 & 60,86 & 68,20 & 50,13 & 63,43 \\
\cline { 2 - 9 } & 76,16 & 66,63 & 74,83 & 70,46 & 77,46 & 65,83 & 72,23 & 58,53 \\
\hline \multirow{4}{*}{ Fibralux } & 17,64 & 37,90 & 0 & 30,93 & 0 & 20,56 & 0 & 11,83 \\
\cline { 2 - 9 } & 20,73 & 38,30 & 15,90 & 31,30 & 0 & 20 & 0 & 11,20 \\
\cline { 2 - 9 } & 16,96 & 38,30 & 0 & 31,20 & 0 & 20,60 & 0 & 11,86 \\
\hline \hline
\end{tabular}


SANTOS, L. A.; TURBINO, M. L; YOUSSEF, M. N.; MATSON, E. Microdureza de resina composta: efeito de aparelhos e tempos de polimerização em diferentes profundidades. Pesq Odont Bras, v. 14, n. 1, p. 65-70, jan./mar. 2000.

TABELA 2 - Análise de Variância - valores originais.

\begin{tabular}{l|r|r|r|r|r}
\hline \hline \multicolumn{1}{c|}{$\begin{array}{c}\text { Fonte de } \\
\text { variação }\end{array}$} & $\begin{array}{c}\text { Soma } \\
\text { quadrados }\end{array}$ & G.L. & $\begin{array}{c}\text { Quadr. } \\
\text { médios }\end{array}$ & F & $\begin{array}{l}\text { Prob } \\
\text { H0 }\end{array}$ \\
\hline Entre tempos & 780,05 & 1 & 780,05 & 6,78 & 2,19 \\
\hline Entre aparelhos & $33.111,78$ & 2 & $16.555,89$ & 144 & 0 \\
\hline Interação T $\times$ A & $1.589,31$ & 2 & 794,65 & 6,91 & 1,01 \\
\hline Resíduo I & $1.379,63$ & 12 & 114,96 & & \\
\hline $\begin{array}{l}\text { Entre } \\
\text { profundidades }\end{array}$ & $4.033,41$ & 3 & 13,44 & 104,92 & 0 \\
\hline Interação P $\times \mathrm{T}$ & 5,13 & 3 & 1,71 & 0,13 & 6,13 \\
\hline Interação P $\times \mathrm{A}$ & 272,70 & 6 & 45,45 & 3,55 & 0,74 \\
\hline $\begin{array}{l}\text { Interação } \\
\text { P } \times \text { T } \times \text { A }\end{array}$ & 245,92 & 6 & 40,98 & 3,20 & 1,27 \\
\hline Resíduo II & 461,29 & 36 & 12,81 & & \\
\hline Variação total & $41.879,28$ & 71 & & & \\
\hline \hline
\end{tabular}

TABELA 3 - Microdureza Knoop de resina composta: interação Aparelho versus Tempo.

\begin{tabular}{l|c|c|c}
\hline \hline \multirow{2}{*}{ Aparelho } & \multicolumn{2}{|c|}{ Tempo } & \multirow{2}{*}{ Tukey 5\% } \\
\cline { 2 - 3 } & $20 \mathrm{~s}$ & $40 \mathrm{~s}$ & \\
\hline Optilight II & 54,64 & 51,76 & \multirow{2}{*}{14,70} \\
\hline XL 1500 & 64,59 & 67,82 & \multirow{2}{*}{ Fibralux } \\
\hline \hline
\end{tabular}

cabo Fibralux (Dabi Atlante) promoveu a menor microdureza. Pelo teste de Tukey $(\mathrm{T}=8,25)$ para a comparação entres as médias pode-se verificar que os aparelhos tipo revólver apresentaram dureza maior em relação ao aparelho a cabo.

Para analisar as profundidades de polimerização, também foi realizado o teste de Tukey $(\mathrm{T}=3,22)$ onde pudemos observar que o primeiro milimetro de profundidade mostrou sempre a maior microdureza.

A visualização e a comparação dos valores obtidos ficam mais evidentes quando se faz a análise da interação dos diferentes fatores.

Para a interação Aparelho versus Tempo (Tabela 3 e Gráfico 1) onde Tukey = 14,7, pode-se observar que só foi detectada diferença entre os aparelhos do tipo revólver no tempo de $40 \mathrm{~s}$, ou seja, o aparelho XL 1500 (3M) promoveu maior dureza ( $\mathrm{m}=67,82)$ que o Optilight II (Gnatus) $(\mathrm{m}=51,76)$.

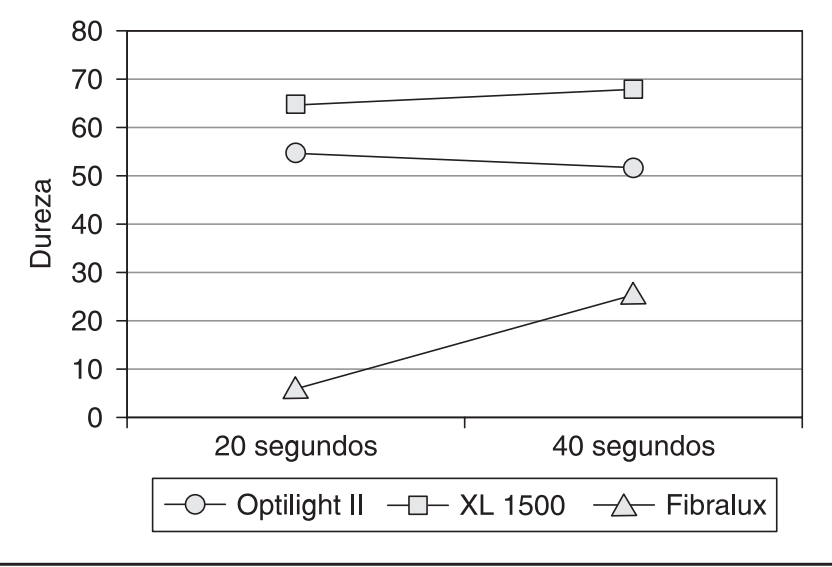

GRÁFICO 1 - Interação Aparelho versus Tempo.

O aparelho a cabo mostrou valores muito baixos, para os dois tempos $(20 \mathrm{~s}=5,93$ e $40 \mathrm{~s}=25,33)$ que foi sempre menor que os outros valores obtidos.

Para a interação Tempo versus Profundidade (Tukey $=5,43$ ) pode-se verificar que numa mesma profundidade a dureza era sempre é maior com 40 s (Tabela 4 e Gráfico 2).

Pode-se observar ainda que no tempo de $40 \mathrm{~s}$ os valores obtidos para determinada profundidade são sempre semelhantes aos valores obtidos em $1 \mathrm{~mm}$ a menos de profundidade, quando a resina foi polimerizada por $20 \mathrm{~s}$.

Para a interação Aparelho versus Profundidade (Tukey $=7,22$ ) podemos observar que nas diferentes profundidades, o aparelho $3 \mathrm{M}$ proporcionou maior dureza que o da Gnatus e esses dois induziram a dureza maior que o aparelho a cabo (Tabela 5 e Gráfico 3).

\section{DISCUSSÃO}

É evidente que a evolução dos aparelhos fotopolimerizadores para o tipo revólver foi significativa. Os nossos resultados confirmam claramente essa afirmação, visto que o aparelho a cabo utilizado sempre levou a graus de dureza muito baixos quando comparados aos demais.

Porém, hoje, o comércio de aparelhos vem se tornando amplo e muito embasado na maior intensidade de luz.

Verifica-se na literatura que as pesquisas recomendam intensidade de luz superior a $233 \mathrm{~mW} / \mathrm{cm}^{2}$ (RUEGGBERG et al. $\left.{ }^{17}, 1994\right) \mathrm{e}$ $300 \mathrm{~mW} / \mathrm{cm}^{2}$ pelos fabricantes do Demetron; entretanto, no presente trabalho, verifica-se para melhores resultados de dureza, intensidade de luz superior a $400 \mathrm{~mW} / \mathrm{cm}^{2}$. 
SANTOS, L. A.; TURBINO, M. L; YOUSSEF, M. N.; MATSON, E. Microdureza de resina composta: efeito de aparelhos e tempos de polimerização em diferentes profundidades. Pesq Odont Bras, v. 14, n. 1, p. 65-70, jan./mar. 2000.

TABELA 4 - Microdureza Knoop de resina composta: interação Tempo versus Profundidade.

\begin{tabular}{c|c|c|c|c|c}
\hline \hline \multirow{2}{*}{ Tempo } & \multicolumn{4}{|c|}{ Profundidade } & Tukey \\
\cline { 2 - 5 } & $1 \mathrm{~mm}$ & $2 \mathrm{~mm}$ & $3 \mathrm{~mm}$ & $4 \mathrm{~mm}$ & $5 \%$ \\
\hline 20 segundos & 51,73 & 45,15 & 38,89 & 31,13 & \multirow{2}{*}{5,43} \\
\hline 40 segundos & 57,44 & 52,30 & 45,68 & 37,80 & \\
\hline \hline
\end{tabular}

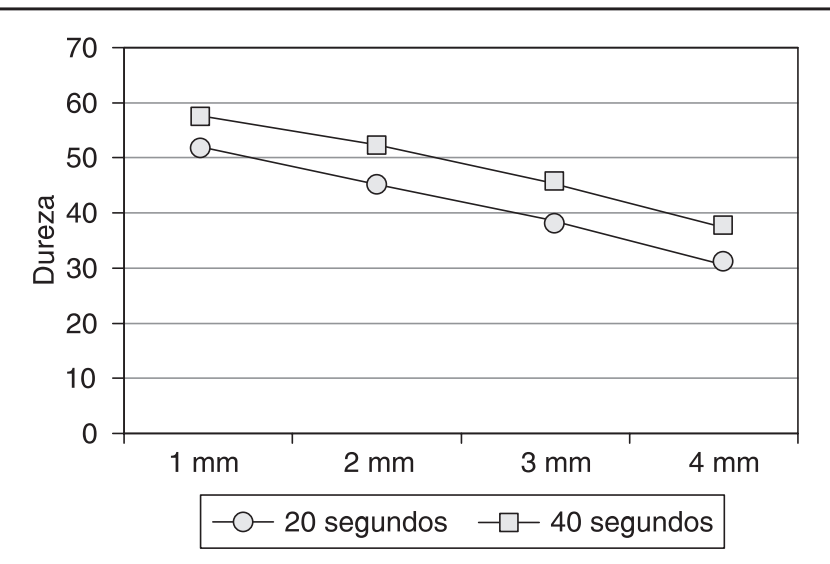

GRÁFICO 2 - Interação Tempo versus Profundidade.

BARGHI et al. ${ }^{3}$ (1994) pode observar que em 205 consultórios, 30\% tinham unidades fotopolimerizadoras insuficientes, (inferiores a $\left.200 \mathrm{~mW} / \mathrm{cm}^{2}\right)$.

Os aparelhos tipo pistola utilizados nesta pesquisa apresentavam sempre valores de intensidade em torno de $400 \mathrm{~mW} / \mathrm{cm}^{2}$.

O controle da qualidade da intensidade de luz fornecida pelos aparelhos deve ser cuidadoso e, com a introdução dos aparelhos radiômetros isso tornou-se mais fácil. Já existem no mercado até aparelhos com um radiômetro embutido na sua base, o que possibilita ao profissional uma averiguação constante do mesmo.

O grau de polimerização da resina composta muitas vezes é avaliado pela microdureza e esta está associada à intensidade de luz dos aparelhos fotopolimerizadores. Entretanto, nem sempre o aparelho com maior intensidade de luz é o de melhor qualidade, visto que outros parâmetros devem ser avaliados.

Os resultados da relação direta entre intensidade de luz e profundidade de polimerização em resinas compostas fotopolimerizáveis são concordantes com os achados de FRIEDMAN; HASSAN ${ }^{12}$ (1984); FAN et al. ${ }^{10}$ (1987); FOWLER et al. ${ }^{11}$ (1994); RUEGGEBER et al. ${ }^{17}$ (1994).
TABELA 5 - Microdureza Knoop de resina composta: interação Profundidade versus Aparelho.

\begin{tabular}{|c|c|c|c|c|c|}
\hline \multirow{2}{*}{ Aparelho } & \multicolumn{4}{|c|}{ Profundidade } & \multirow{2}{*}{$\begin{array}{c}\text { Tukey } \\
5 \%\end{array}$} \\
\hline & $1 \mathrm{~mm}$ & $2 \mathrm{~mm}$ & $3 \mathrm{~mm}$ & $4 \mathrm{~mm}$ & \\
\hline Optilight II & 63,46 & 57,72 & 51,03 & 40,58 & \multirow{3}{*}{7,22} \\
\hline XL 1500 & 71,99 & 70,23 & 65,64 & 57,00 & \\
\hline Fibralux & 28,30 & 18,22 & 10,19 & 5,81 & \\
\hline
\end{tabular}

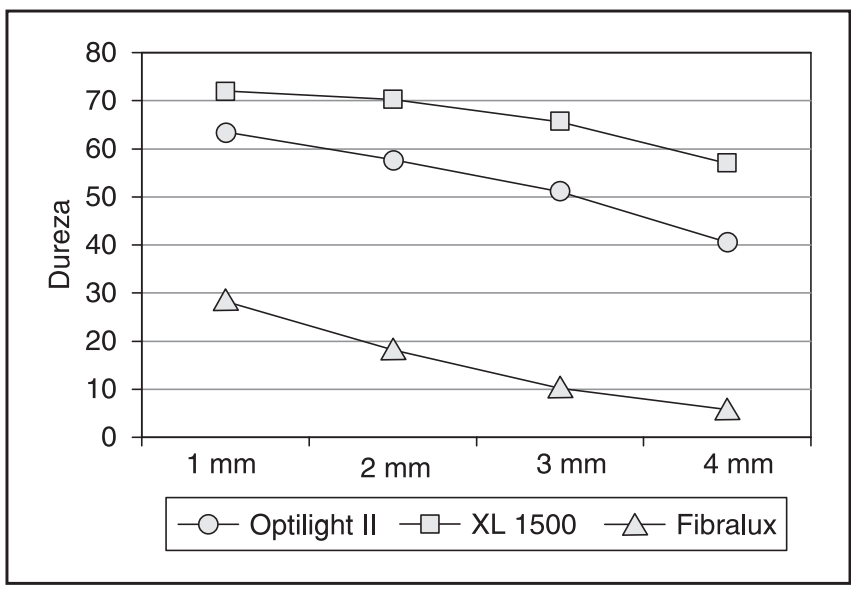

GRÁFICO 3 - Interação Aparelho versus Profundidade.

Segundo COOK; STANDISH ${ }^{6}$ (1983); NEWMAN et $a l .{ }^{15}$ (1983), a profundidade de polimerização é influenciada por fatores tais como: intensidade de luz e tempo de polimerização.

O tempo de polimerização influencia na dureza do material. Isso foi observado no estudo de BROSH et al..$^{5}$ (1997) que fotopolimerizaram resinas compostas com os tempos de 20, 40, 60 e $180 \mathrm{~s}$.

Clinicamente, a profundidade de polimerização da resina composta é importante, visto que em cavidades amplas, a inserção do material deve ser realizada sempre em incrementos (DE GEE et al. ${ }^{8}$, 1981). Baseados em nossos resultados o primeiro milimetro de profundidade foi o que apresentou a maior microdureza, principalmente quando com tempo de $20 \mathrm{~s}$. Para o tempo de $40 \mathrm{~s}$ a dureza dos 2 primeiros milimetros foi semelhante porém, do terceiro $\mathrm{mm}$ em diante, a dureza já era reduzida. Com isso, podemos sugerir que os incrementos de resina composta não devem ter espessura superior a $2 \mathrm{~mm}$ quando se usa um tempo de $40 \mathrm{~s}$. Porém com tempo de 20 s essa espessura máxima passa a ser de $1 \mathrm{~mm}$.

SHORTALL; HARRIGTON ${ }^{18}$ (1996) usando três resinas de marcas diferentes e vinte luzes percebe- 
SANTOS, L. A.; TURBINO, M. L; YOUSSEF, M. N.; MATSON, E. Microdureza de resina composta: efeito de aparelhos e tempos de polimerização em diferentes profundidades. Pesq Odont Bras, v. 14, n. 1, p. 65-70, jan./mar. 2000.

ram uma significante correlação entre a dureza a $2 \mathrm{~mm}$ de profundidade e a intensidade de luz. DUNNE et al. ${ }^{9}$ (1996) verificaram que com baixa intensidade de luz a resina composta apenas tinha aparência mas não podia ser considerada totalmente polimerizada. Conforme aumentava o tempo de polimerização de 20 para $60 \mathrm{~s}$, aumentava a profundidade da resina polimerizada.

HANSEN; ASMUSSEN ${ }^{14}$ (1997) usaram profundidades de restaurações proximais para verificar sua microdureza em $0,5,1,0,1,5$ e $2,0 \mathrm{~mm}$. Concluíram, como esperado, que a cada $0,5 \mathrm{~mm}$ decresce moderadamente sua microdureza.

A inserção e fotopolimerização da resina composta em incrementos também está associada à contração de polimerização, visto que essa contração é proporcional ao volume de material (DAVIDSON; DE GEE 7 , 1984).

GORACCI et al. ${ }^{13}$ (1996) ainda correlacionaram a contração de polimerização com a intensidade de luz. Verificaram que ao utilizar alta intensidade desde o início da polimerização, a contração era maior do que quando essa intensidade era aumentada gradativamente.

\section{CONCLUSÕES}

Consideramos que:

1. para o tempo de $40 \mathrm{~s}$ de polimerização a microdureza obtida foi maior que com $20 \mathrm{~s}$ para os três aparelhos;

2. os aparelhos fotopolimerizadores do tipo pistola (XL 1500 e Optilight II), promoveram maior microdureza na resina composta que o aparelho tipo cabo Fibralux (Dabi Atlante), sendo que o aparelho XL 1500 (3M) no tempo de $40 \mathrm{~s}$ induziu valores maiores de microdureza que o Optilight II (Gnatus);

3. as profundidades de 1, 2, 3 e 4 mm mostraram diferenças estatisticamente significantes entre si, tendo sido encontrada maior dureza para as menores profundidades.

SANTOS, L. A.; TURBINO, M. L; YOUSSEF, M. N.; MATSON, E. Microhardness of composite resins: effect of photocuring units and polymerization periods in different depths. Pesq Odont Bras, v. 14, n. 1, p. 65-70, jan./mar. 2000.

\begin{abstract}
The properties of composite resins have been frequently studied, as well as the factors that might influence the polymerization degree. Considering the evolution of composites and the need to improve their clinical performance, this study intends to evaluate the efficacy of two high-intensity and one low-intensity photocuring units, as a function of exposure time (20 and 40seconds) and composite depth (from 1 to 4 millimeters). Composite properties were evaluated via Knoop Microhardness Test. Statistical analysis (1 way ANOVA, p < 0.05) of the obtained results showed that there was statistically significant difference regarding the time factor. The 40-seconds polymerization time showed the best results, for the four depths. Concerning the photocuring unit factor, there was statistically significant (1- way ANOVA, $\mathrm{p}<0.05)$ difference between the tested devices. The high-intensity units demonstrated better results than the low-intensity one. There were statistically significant differences between the analysed composite depths, being the highest microhardness values achieved for the lowest depths.
\end{abstract}

UNITERMS: Composite resins; Dental materials; Hardness.

\section{REFERÊNCIAS BIBLIOGRÁFICAS}

1. AMERICAN DENTAL ASSOCIATION. Council on Dental Material, Instruments and Equipment. Visible light-cured composite and activating units. J Am Dent Assoc, v. 110, n. 1-3, p. 100-103, 1985.

2. ARAÚJO, R. M.; ARAÚJO, M. A. M.; MEndES, A. J. D. Influência da intensidade de luz de fotopolimerizadores na infiltração marginal. Rev Assoc Paul Cir Dent, v. 50, n. 5, p. 408-413, 1996.

3. BARGHI, N.; BERRY, T.; HATTON, N.C. Evaluating intensity out put of curing lights. J Am Dent Assoc, v. 125, n. 7-9, p. 992-996, 1994

4. BASSIOUNY, M. A.; GRANT, A. A. Physical properties of a visible light-cured composite resin. J Prosthet Dent, v. 43, n. 5, p. 536-545, 1980.

5. BROSH, T.; BAHARAU, H.; GROSS, O.; LAUFER, B. Z. The influence of surface loading and irradiation time during curing on mechanical properties of a composite. $\mathbf{J}$ Prosthet Dent, v. 77, n. 6, p. 573-577, 1997.

6. COOK, W. D.; STANDISH, P.M. Cure of resin based restorative materials II. White light photopolimerized resins.

Aust Dent J, v. 28, n. 5, p. 307-311, 1983.

7. DAVIDSON, C. L.; DE GEE, A. J. Relation of polymerization contraction stresses by flow in dental composite. J Dent Res, v. 63, n. 1-6, p. 146-148, 1984.

8. DE GEE, A. J.; DAVIDSON, A.; SMITH A. A. Modified dilatatomer of continuous recording of volumetric polymerization shrinkage of composite restorative materials. $\mathbf{J}$ Dent, v. 9, p. 36-42, 1981.

9. DUNNE, S. M.; DAVIES, B. R.; MILLAR, B. J. A survey of the effectiveness of dental light-curing units and a com- 
SANTOS, L. A.; TURBINO, M. L; YOUSSEF, M. N.; MATSON, E. Microdureza de resina composta: efeito de aparelhos e tempos de polimerização em diferentes profundidades. Pesq Odont Bras, v. 14, n. 1, p. 65-70, jan./mar. 2000.

parison of light testing devices. Br Dent J, v. 180, n. 11, p. 411-416, 1996.

10. FAN, P. L.; WOZNIAK, W. T.; REYES, W. D.; STANFORD, J. W. Irradiance of visible lights-curing units and voltage variation effects. J Am Dent Assoc, v. 115, n. 1-3, p. 442-445, 1987.

11. FOWLER, C. S.; SWARTZ, M. L.; MOORE, B. K. Efficacy testing of visible light-curing units. Oper Dent, v. 19, n. 2, p. 47-52, 1994.

12. FRIEDMAN, J.; HASSAN, R. Comparative study of visible curing lights and hardness of light-cured restorative materials. J Prosthet Dent, v. 52, n. 4-6, p. 504-506, 1984.

13. GORACCI, G.; MORI, G.; MARTINS, L.C. Curing light intensity and marginal leakage of resin composite restorations. Quintessence Int, v. 27, n. 5, p. 355-367, 1996.

14. HANSEN, E. K.; ASMUSSEN, E. Visible light-curing units: correlation between depht of cure and distance between exit window and resin surface. Acta Odontol Scand, v. 55, n. 3, p. 162-166, 1997.
15. NEWMAN, S. M.; MURRAY, G. A.; YATES, J.L. Visible lights and visible light-activated resin composites. J Prosthet Dent, v. 50, n. 1, p. 31-35, 1983.

16. PILO, R.; CARDASH, H. S. Pos irradiation polymerization of different interior and posterior visible light-activated resin composites. Dent Mater, v. 8, n. 5, p. 299-304, 1992.

17. RUEGGBERG, F. A.; CAUGHMAN, W. F.; CURTIS, J. W. Jr. Effect of light intensity and exposure duration on cure of resin composite. Oper Dent, v. 19, n. 1-3, p. 26-32, 1994.

18. SHORTAL, A. C.; HARRINGTON, E. Effect of light intensity on polymerization of three composite resins. Eur $\mathbf{J}$ Prosthodont Restor Dent, v. 4, n. 2, p. 71-76, 1996.

19. TURBINO, M. L.; VINHA, D.; CENTOLA, A. L. B., CAMPOS, G. M. Photopolimerized resins. Surface hardness variation in relation to time of polimerization and setting. Braz Dent J, v. 3, n. 3, p. 87-94, 1992.

Recebido para publicação em 25/10/99 Enviado para reformulação em 02/12/99 Aceito para publicação em 17/02/00 